\title{
Endogenous DKK1 and FRZB Regulate Chondrogenesis and Hypertrophy in Three-Dimensional Cultures of Human Chondrocytes and Human Mesenchymal Stem Cells
}

\author{
Leilei Zhong, ${ }^{1, *}$ Xiaobin Huang, ${ }^{1, *}$ Emilie Dooms Rodrigues, ${ }^{1}$ Jeroen C.H. Leijten, \\ Theo Verrips, Mohamed El Khattabi, Marcel Karperien,, and Janine N. Post ${ }^{1}$
}

Hypertrophic differentiation occurs during in vitro chondrogenesis of mesenchymal stem cells (MSCs), decreasing the quality of the cartilage construct. Previously we identified WNT pathway antagonists Dickkopf 1 homolog (DKK1) and frizzled-related protein (FRZB) as key factors in blocking hypertrophic differentiation of human MSCs (hMSCs). In this study, we investigated the role of endogenously expressed DKK1 and FRZB in chondrogenesis of hMSC and chondrocyte redifferentiation and in preventing cell hypertrophy using three relevant human cell based systems, isolated hMSCs, isolated primary human chondrocytes (hChs), and cocultures of hMSCs with hChs for which we specifically designed neutralizing nano-antibodies. We selected and tested variable domain of single chain heavy chain only antibodies $(\mathrm{VHH})$ for their ability to neutralize the function of DKK1 or FRZB. In the presence of DKK1 and FRZB neutralizing VHH, glycosaminoglycan and collagen type II staining were significantly reduced in monocultured hMSCs and monocultured chondrocytes. Furthermore, in cocultures, cells in pellets showed hypertrophic differentiation. In conclusion, endogenous expression of the WNT antagonists DKK1 and FRZB is necessary for multiple steps during chondrogenesis: first DKK1 and FRZB are indispensable for the initial steps of chondrogenic differentiation of hMSCs, second they are necessary for chondrocyte redifferentiation, and finally in preventing hypertrophic differentiation of articular chondrocytes.

Keywords: cell signaling, chondrogenesis, differentiation, MSC

\section{Introduction}

A RTICULAR CARTILAGE REPAIR is a challenge due to very limited capacity of self-repair after damage. Autologous chondrocyte implantation (ACI) has become the golden standard treatment for large-size cartilage defects [1]. However, ACI leads to donor-site morbidity and is dependent on 2D expansion of isolated chondrocytes, which are prone to undergo dedifferentiation during proliferation [2]. To overcome this drawback, a (partial) replacement of chondrocytes with mesenchymal stem cells (MSCs) is an option. Although the application of MSCs in cartilage repair is promising, the use of MSCs in cartilage engineering still has some drawbacks. One of the problems with using MSCs for cartilage regeneration is the expression of hypertrophic markers during in vitro chondrogenesis followed by formation of transient calcifying cartilage [3].

So maintaining the chondrogenic phenotype during in vitro expansion and avoiding hypertrophy of chondrogenically differentiating MSCs remain a big challenge in these cell-based strategies [4]. Successful chondrogenesis of human MSCs (hMSCs) is regulated by various signaling pathways, including FGF, TGF- $\beta$, WNT/ $\beta$-catenin, Notch, and hypoxia [5]. Articular chondrocytes produce a stable nonmineralizing cartilage [6], and coculture of MSCs and articular cartilage reduces hypertrophy [7] and enhances functional properties of engineered cartilage [8].

$\mathrm{WNT} / \beta$-catenin signaling plays a pivotal role in articular cartilage [9], where enhanced signaling activity results in cartilage destruction through chondrocyte hypertrophic

\footnotetext{
${ }^{\mathrm{l}}$ Department of Developmental BioEngineering, Faculty of Science and Technology, MIRA-Institute for Biomedical Technology and Technical Medicine, University of Twente, Enschede, the Netherlands.

${ }^{2}$ QVQ BV, Utrecht, the Netherlands.

*These authors contributed equally to this work.

(C) Leilei Zhong, et al., 2016; Published by Mary Ann Liebert, Inc. This Open Access article is distributed under the terms of the Creative Commons Attribution Noncommercial License (http://creativecommons.org/licenses/by-nc/4.0/) which permits any noncommercial use, distribution, and reproduction in any medium, provided the original author(s) and the source are credited.
} 
differentiation and subsequent development of an osteoarthritis (OA)-like phenotype [10]. Decreased WNT activity also results in cartilage destruction through the induction of apoptosis [11]. Therefore, maintaining strict control over WNT/ $\beta$-catenin signaling in articular cartilage is of the highest importance.

We previously found that the WNT antagonists Dickkopf 1 homolog (DKK1) and frizzled-related protein (FRZB) are highly enriched in articular cartilage compared to epiphyseal growth plate cartilage [12]. These antagonists constitute a key part of the natural mechanism that balances $\mathrm{WNT} / \beta$ catenin signaling activity. DKK1 antagonizes WNT signaling pathway by binding to the frizzled coreceptors' low-density lipoprotein receptor-related protein 5 (LRP-5) and LRP-6 $[13,14]$. Secreted frizzled-related proteins (sFRP) antagonize WNT signaling through interactions with WNTs and/or through the formation of nonfunctional complexes with the frizzled receptors [15-17], resulting in the inhibition of both canonical and noncanonical pathways [18]. DKK1 increases hMSC proliferation and promotes entry into the cell cycle in vitro [19]. In addition, we have previously shown that in chondrogenically differentiating hMSC pellet cultures and in explanted mouse tibiae, exogenous addition of DKK1 or FRZB prevented terminal hypertrophic differentiation of chondrocytes, supporting their role in articular cartilage homeostasis [12].

WNT pathway activity repressed the expression of cartilage-specific extracellular matrix (ECM) molecules and might be involved in chondrocyte dedifferentiation during in vitro expansion of primary human chondrocytes (hChs) [20,21]. Healthy articular chondrocytes keep their differentiated state and do not undergo dedifferentiation suggesting that endogenous WNT/ $\beta$-catenin signaling is blocked by an unknown mechanism. DKK1 and FRZB are natural antagonists and are highly expressed by healthy hChs [12]. We previously found that when cultured under prolonged passaging, chondrocytes lose their phenotype and that this is accompanied by changes in expression of DKK1 and FRZB [22]. This indicates that the combined endogenous expression of DKK1 and FRZB plays a role in chondrocyte dedifferentiation.

So the correct regulation of the WNT signaling plays an important role in the maintenance of the chondrogenic potential, as well as in suppression of endochondral ossification [23]. However, the mechanism by which WNT signaling is regulated in vivo is as yet unknown. It has been reported that FRZB knockout mice have no cartilage phenotypic changes, but are more susceptible to OA in mechanical injury [24]. DKK1 knockout mice show no change in cartilage degeneration; only osteophyte formation is increased upon mechanical loading [24]. This suggests the functional redundancy of DKK1 and FRZB because both of them are capable of inhibiting the WNT/ $\beta$-catenin pathway, one can compensate for the loss of the other. We propose that the combined endogenous expression of DKK1 and FRZB plays a determining role in both the chondrogenesis of hMSCs and prevention of hypertrophic differentiation in vivo.

There is ample evidence that the correct modulation of WNT activity is necessary and sufficient for the formation and maintenance of healthy articular cartilage. However, the exact mechanisms regulating the amplitude of WNT activity in articular cartilage in vivo are as yet unknown and are the subject of this project. We hypothesized that endogenous expression of DKK1 and FRZB is both necessary and suffi- cient for articular cartilage formation and prevention of hypertrophic differentiation. Specifically, we hypothesized that simultaneously blocking DKK1 and FRZB would negatively influence the chondrogenic potential of hMSCs and dedifferentiated chondrocytes and that blocking endogenous DKK1 and FRZB leads to hypertrophic differentiation. To prove our hypotheses, we have evaluated the combined role of endogenous levels of DKK1 and FRZB by blocking their activity using neutralizing $\mathrm{VHH}$ antibodies in three relevant human cell based systems: isolated hMSCs, isolated primary hChs, and cocultures of hMSCs with hChs.

\section{Materials and Methods}

\section{Selection of anti-DKK1 VHH from immune VHH library}

Two llamas were immunized with recombinant human DKK1 (R\&D Systems) as described in reference 25. The immunization protocol was approved by the Utrecht University Ethics Committee (DEC: 2007.III.01.013). Total RNA was extracted from peripheral blood lymphocytes; cDNA was synthesized. VHH were subsequently cloned in phagemid pUR8100, which was derived from pHEN [26] described by reference 27 . VHH binding to DKK1 was selected by panning in two rounds of selection as described [25]. Screening of the selected $\mathrm{VHH}$ for binding to DKK1 led to the identification of 14 different VHH. The amino acid sequences of the VHH show different clones from different families (Supplementary Fig. S1; Supplementary Data are available online at www.liebertpub .com/scd), of which two (G5 and H7) were recurrent and, therefore, used for further analysis.

To test the affinity of the anti-DKK1 VHH, sequences were subcloned into the expression plasmid pMEK219 containing $\mathrm{C}$ terminal Myc and His tags. VHH were subsequently produced from Escherichia coli by induction of the lac promoter with $1 \mathrm{mM}$ isopropyl $\beta$-D-1-thiogalactopyranoside [28,29] and were purified from the periplasmic fraction through the $\mathrm{C}$ terminal His-tag by cobalt affinity chromatography (TALON His-Tag Purification Resin; Clontech). The size and purity of the VHH were assessed by SDS-PAGE [30,31].

Purified VHH were tested by enzyme-linked immunosorbent assay (ELISA). Plates were coated with DKK1 $(60 \mathrm{nM})$ and blocked with $4 \%$ skimmed milk in phosphatebuffered saline (MPBS), then incubated with a concentration range of $\mathrm{VHH}(0-7 \mu \mathrm{M})$. Unbound $\mathrm{VHH}$ were washed with PBS-Tween (PBST), and bound VHH were detected by incubation with $\mathrm{mAb}$ anti-myc (9E10) and a horseradish peroxidase-conjugated anti-mouse.

To assess the biological activity of the anti-DKK1 VHH, KS483-4C3 mouse progenitor cells were used as a model for osteogenic differentiation [32]. Cells were seeded at 10,000 cells $/ \mathrm{cm}^{2}$ (day 0). At day 4, cells were cultured for another 3 days with ascorbic acid $(50 \mu \mathrm{g} / \mathrm{mL}$; Sigma Aldrich) and stimulated with BMP6 (100 ng/mL; R\&D Systems) in the presence or absence of DKK1 (300 ng/mL; R\&D Systems) with a concentration series of VHH G5 or H7 (0-70 nM). At day 7, alkaline phosphatase (ALP) activity was evaluated by CDP-Star Kit (Roche). Luminescence was measured using Vector Microplate Luminometer (Promega). The luminescence units were corrected for DNA content. DNA concentration was determined using the CyQUANT Cell Proliferation Assay (Invitrogen). 


\section{Selection of anti-FRZB from a nonimmunized Ilama VHH library}

VHH binding to FRZB (R\&D Systems) was selected from nonimmunized llama VHH-phage display library [33], kindly provided by BAC B.V. (Thermo Fisher) in two panning rounds [33]. Selection and screening were as described for the antiDKK1 VHH, with the exception of applying more phages for the first round of selection [33]. Screening of the FRZB binders led to identification of five VHH candidates. The amino acid sequences of the VHH are indicated in Supplementary Fig. S2. Anti-FRZB VHH were cloned in the expression plasmid pMEK222 containing $\mathrm{C}$ terminal FLAG and His tags. Production and purification of the $\mathrm{VHH}$ were as described for the anti-DKK1 VHH. Apparent affinity of the purified FRZB VHH was measured with ELISA as described for anti-DKK1, with the exception of detecting bound VHH with mAb M1 directed against FLAG instead of mAb 9E10.

\section{Cell culture and expansion}

Human primary chondrocytes were obtained from relatively healthy looking full thickness cartilage, dissected from knee biopsies of three patients [mean \pm standard deviation (SD) age $60 \pm 3$ years] undergoing total knee replacement, as described previously [34]. To isolate cells, the cartilage was digested in chondrocyte proliferation medium containing collagenase type II (0.15\%; Worthington) for 20-22 h. Subsequently, the hChs were expanded at a density of 3,000 cells $/ \mathrm{cm}^{2}$ in chondrocyte proliferation medium until the monolayer reached $80 \%$ confluency. Chondrocyte proliferation medium consisted of Dulbecco's modified Eagle's medium (DMEM) supplemented with $10 \%$ fetal bovine serum (FBS), $1 \times$ nonessential amino acids, $0.2 \mathrm{mM}$ ascorbic acid 2-phosphate (AsAP), $0.4 \mathrm{mM}$ proline, $100 \mathrm{U} / \mathrm{mL}$ penicillin, and $100 \mu \mathrm{g} / \mathrm{mL}$ streptomycin. The hChs were used in passage two unless otherwise stated. The hMSCs were isolated from human bone marrow aspirates as described previously [34] and cultured in MSC proliferation medium $(\alpha$-MEM supplemented with 10\% FBS, 1\% Lglutamax, $0.2 \mathrm{mM}$ ascorbic acid, $100 \mathrm{U} / \mathrm{mL}$ penicillin, $100 \mu \mathrm{g} /$ $\mathrm{mL}$ streptomycin, and $1 \mathrm{ng} / \mathrm{mL}$ bFGF).

\section{Pellet cultures and chondrogenic differentiation}

To get enough cell pellets for analysis, micropatterned agarose chips were used in this experiment. Micropatterned agarose chips were prepared at a concentration of $4 \% \mathrm{w} / \mathrm{v}$ by replica molding as described previously [35]. Polydimethylsiloxane stamps were used to routinely replicate the microstructures. For monocultures, 250,000 cells of hChs or hMSCs were seeded into 1,585 microwells of micropatterned agarose chips. This resulted in roughly 160 cells/pellet.

For cocultures, 250,000 cells were seeded at a ratio of hMSC/ $\mathrm{hChs}=80 / 20$. Cells were suspended in chondrogenic differentiation medium [DMEM supplemented with $50 \mu \mathrm{g} / \mathrm{mL}$ ITSpremix, $50 \mu \mathrm{g} / \mathrm{mL}$ of AsAP, $100 \mu \mathrm{g} / \mathrm{mL}$ of sodium pyruvate, $10 \mathrm{ng} / \mathrm{mL}$ of TGF- $\beta 3,10^{-7} \mathrm{M}$ of dexamethasone (DEX), $100 \mathrm{U} / \mathrm{mL}$ of penicillin, and $100 \mu \mathrm{g} / \mathrm{mL}$ of streptomycin] and pipetted into the agarose wells. Subsequently, the agarose chips were centrifuged for $5 \mathrm{~min}$ at $500 \mathrm{~g}$ to form pellets. Cell pellets were incubated in the microwells at $37^{\circ} \mathrm{C}$ in a humid atmosphere with $5 \% \mathrm{CO}_{2}$ for a period of 4 weeks before analysis. The medium was supplemented with $5 \mu \mathrm{g} / \mathrm{mL}$ of VHH anti-DKK1 (D-H7) and VHH anti-FRZB (TSF-1F7). Nonspecific goat IgG $(5 \mu \mathrm{g} / \mathrm{mL})$ was used as a negative control. The medium, with antibody supplements, was refreshed twice per week.

\section{Total RNA extraction and quantitative polymerase chain reaction}

Cell pellets were pooled and RNA was isolated using the NucleoSpin RNA II Kit (Macherey-Nagel, Duren, Germany). The concentration and purity of RNA samples were determined using the NanoDrop 2000 (Thermo scientific, Wilmington, United States). Total mRNA was reverse-transcribed into cDNA using the iScript cDNA Synthesis Kit (Bio-Rad, Hercules, CA). Quantitative polymerase chain reaction (qPCR) was performed using the SYBR Green SensiMix (Bioline). PCR was carried out using the Bio-Rad CFX96 (Bio-Rad) under the following conditions: cDNA was denatured for $5 \mathrm{~min}$ at $95^{\circ} \mathrm{C}$, followed by 39 cycles consisting of $15 \mathrm{~s}$ at $95^{\circ} \mathrm{C}, 15 \mathrm{~s}$ at $60^{\circ} \mathrm{C}$, and $30 \mathrm{~s}$ at $72^{\circ} \mathrm{C}$. For each reaction, a melting curve was generated to test primer dimer formation and nonspecific priming. Primer sequences are listed in Supplementary Table S1.

\section{Histological analysis}

Cell pellets were collected after 4 weeks incubation and fixed with $10 \%$ phosphate-buffered formalin $(\mathrm{pH}=7)$ for $15 \mathrm{~min}$ at room temperature, dehydrated with graded ethanol, and embedded in paraffin using routine procedures. Five micrometer thick sections were cut using a microtome (Shandon). Before staining, the slides were deparaffinized in xylene and rehydrated with graded ethanol.

Alcian blue and safranin $O$ staining. Slides were either stained for sulfated glycosaminoglycans (GAGs) with a $0.5 \%$ w/v solution of Alcian blue $(\mathrm{pH}=1$, adjusted with $\mathrm{HCl})$ for $30 \mathrm{~min}$. The samples were then counterstained with nuclear fast red $(0.1 \% \mathrm{w} / \mathrm{v}$ of nuclear fast red in 5\% aluminum sulfate) for 5 min to visualize nuclei [36] or stained for sulfated GAG with a $0.1 \%$ solution of safranin O for $5 \mathrm{~min}$ (Sigma Aldrich).

Alizarin red S staining. Slides were stained with a $2 \% \mathrm{w} / \mathrm{v}$ solution of Alizarin red $\mathrm{S}(\mathrm{pH}=4.2$, adjusted with ammonium hydroxide) to assess the presence of mineralization. Light microscopy was used to visualize red mineralized nodules as described [36].

\section{Immunohistochemistry}

Immunohistochemical staining of collagen type II was performed using $5 \mu \mathrm{m}$ sections. Slides were deparaffinized in xylene and rehydrated with graded ethanol. Samples were preincubated with $5 \mu \mathrm{g} / \mathrm{mL}$ proteinase K (Sigma Aldrich) for $10 \mathrm{~min}$ at room temperature followed by $1 \mathrm{mg} / \mathrm{mL}$ hyaluronidase (Sigma Aldrich) for $40 \mathrm{~min}$ at $37^{\circ} \mathrm{C}$. Samples were blocked in $1.5 \%$ normal goat blocking serum in PBS for $1 \mathrm{~h}$. Rabbit polyclonal collagen type II antibody (Abcam; ab34712) was diluted 1:200 in PBS containing 1.5\% blocking serum (Santa Cruz Biotechnology) and incubated overnight at $4^{\circ} \mathrm{C}$. Nonimmune controls underwent the same procedure without primary antibody incubation. The target protein was detected by incubation in rabbit ABC staining system (sc-2018; Santa Cruz) according to the manufacturer's protocol and imaged using a NanoZoomer. 


\section{Immunofluorescent staining}

Immunofluorescent (IF) staining of collagen type II and collagen type $X$ was performed using $5 \mu \mathrm{m}$ sections. Slides were deparaffinized in xylene and rehydrated with graded ethanol. Samples were preincubated with $5 \mu \mathrm{g} / \mathrm{mL}$ proteinase K (Sigma Aldrich) for $10 \mathrm{~min}$ at room temperature followed by $1 \mathrm{mg} / \mathrm{mL}$ hyaluronidase (Sigma Aldrich) for $40 \mathrm{~min}$ at $37^{\circ} \mathrm{C}$. Samples were blocked in 5\% BSA in PBS for $1 \mathrm{~h}$, then incubated with rabbit anti-collagen II antibody (ab34712; Abcam), which was diluted 1:100 in 5\% BSA in PBS and mouse collagen type X (2031501005; Quartett LifeSpan), which was diluted 1:50 overnight at $4^{\circ} \mathrm{C}$. Cells were rinsed with PBST thrice, $5 \mathrm{~min} / \mathrm{time}$. Then Alexa ${ }^{\circledR}$ Fluor 546-labeled goat anti-rabbit or anti-mouse antibody in 5\% BSA in PBS was added and incubated for $2 \mathrm{~h}$ at room temperature. Samples were rinsed with PBS and added mounting medium with DAPI. Slides were viewed by BD pathway confocal microscopy.

\section{Apoptosis assay}

Apoptosis was detected in paraffin-embedded pellets using The DeadEnd ${ }^{\mathrm{TM}}$ Colorimetric TUNEL assay (Promega) following the manufacturer's procedure. Apoptotic nuclei were stained dark brown.

\section{Statistical analysis}

For the experiments using primary human cells (hMSCs and hChs), samples were obtained from three hCh donors and two hMSC donors. Each experiment was performed in triplicate. Statistical differences between two groups were analyzed by two-tailed Student's $t$-tests or one-way ANOVA. $P<0.05$ was considered statistically significant and indicated with an asterisk. Data are expressed as the mean \pm SD.

\section{Results}

The camelidae family expresses a special class of antibodies that are devoid of light chains [37]. These antibodies are called single domain antibodies engineered from heavy chain camelid antibodies ( $\mathrm{VHH}$ ), which are formed by two protein chains. Compared with conventional antibodies, using VHH has several advantages, including small size (15 kDa) and high binding specificity and affinity [38], simple and easy to clone, and subsequently to genetically modify, they can be produced in bacteria and yeast $[38,39]$. Moreover, VHH antibodies easily penetrate into $3 \mathrm{D}$ cell pellets. Selection of functional $\mathrm{VHH}$ is of the utmost importance to determine the quality of the data and is not trivial. We therefore describe the selection of the VHH against DKK1 and the VHH against FRZB in the sections below.

\section{Selection of anti-DKK1 $\mathrm{VHH}$}

Anti-DKK1 VHH were selected from llama VHH-phage display libraries immunized with recombinant DKK1. The phage display library is constructed from RNA of peripheral blood mononuclear cells isolated from the immunized llama. Selection led to the identification of two VHH, G5 and $\mathrm{H} 7$, which display apparent affinities $(\mathrm{Kd})$ of $5.9 \times 10^{-8}$ $\mathrm{M}$ and $1.1 \times 10^{-7} \mathrm{M}$, respectively (Fig. 1A). The ability of the two VHH to modulate DKK1 biological activity was tested in vitro in murine KS483-4C3 mesenchymal precursor cells, which were induced to express ALP after treatment with BMP6. BMP-induced ALP expression is mediated through canonical WNT-signaling [10]. Indeed cotreatment of cells with BMP6 and DKK1 effectively abrogated ALP induction (Fig. 1B). This inhibition could be reversed by the addition of VHH G5 or H7 in a dosedependent manner (Fig. 1C, D). Both VHH showed effective neutralization of DKK1 in this bioassay.

\section{Selection of anti-FRZB VHH}

VHH against FRZB were selected from a nonimmune llama VHH-phage display library. Two rounds of panning selection led to the selection of eight candidate VHH. After subcloning into pMEK222 and sequence determination, five VHH with different sequences were successfully expressed and purified. ELISA was used to rank the different VHH according to the apparent affinities (Fig. 1E).

\section{Functional analysis of anti-DKK1 and anti-FRZB VHH}

Functional assays were performed to choose the most efficient VHH out of two DKK1 VHH and five FRZB VHH in blocking the function of DKK1 or FRZB in human primary chondrocytes, respectively. We found that $\mathrm{H} 7$ was most efficient in blocking DKK1, and TSF-1F7 was most effective for blocking FRZB (Supplementary Fig. S3A, B). Although TSF-1F7 has the lowest affinity to FRZB based on ELISA, it has the best neutralizing efficiency in cells compared to the other four FRZB VHH. This might be due to specific binding of TSF-1F7 to the biological domain of endogenous FRZB thereby blocking its function, while the other four FRZB VHH bind other FRZB domains.

\section{VHH against DKK1 and FRZB function as neutralizing antibodies as measured by restored WNT signaling}

Dose-response experiments were performed to determine the optimum concentration of the VHH in blocking DKK1 and FRZB. A concentration of $5 \mu \mathrm{g} / \mathrm{mL}$ of neutralizing VHH completely reversed the blocking effect of DKK1 or FRZB on WNT3A-induced AXIN2 mRNA expression as determined by qPCR analysis (Fig. 2A, B). This concentration of $5 \mu \mathrm{g} / \mathrm{mL}$ was used in all subsequent experiments. At the same concentration, a nonspecific IgG control did not have any effect on the expression of the examined marker genes (Fig. 2C-E).

\section{Blocking DKK1 and FRZB inhibits both chondrogenesis of hMSCs and chondrocyte redifferentiation}

We have found that the endogenous expression levels of DKK1 and FRZB in hMSCs are low (Supplementary Fig. S4), while their expression increases during hMSC chondrogenesis (Supplementary Fig. S5). This suggests that DKK1 and FRZB are involved in chondrogenesis of hMSCs. To prove this, neutralizing VHH were added to block DKK1 and FRZB in monoculture hMSC pellets during chondrogenic induction. 

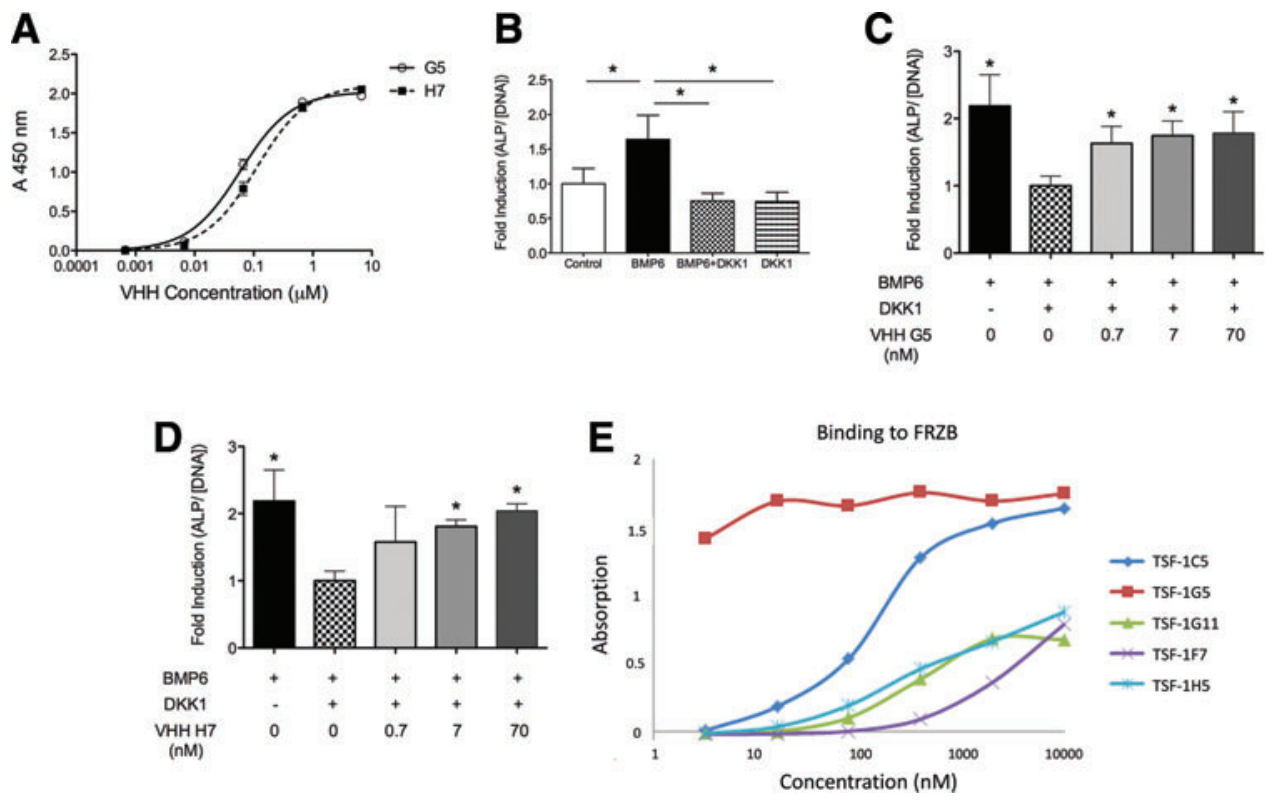

FIG. 1. Selection and analysis of anti-DKK1 and anti-FRZB VHH, (A) VHH bind to DKK1 and neutralize DKK1 activity. Concentration range of VHH G5 and H7 was incubated in wells coated with DKK1 (60 nM) or PBS (negative control). After several washes, bound VHH were detected with a mouse anti-VHH serum and a donkey anti-rabbit antibody coupled to a peroxidase. The amount of converted HRP (absorbance at $450 \mathrm{~nm} ; \mathrm{A} 450 \mathrm{~nm}$ ) is proportional to the amount of bound VHH. Error bars represent SD $(n=3)$. (B) Addition of BMP6 stimulates ALP expression in KS483-4C3 cells. The coincubation of BMP6 with DKK1 reversed the ALP expression back to basal levels. The addition of DKK1 to the standard cell culture did not show any significant difference in ALP expression compared with control. The ALP activity is normalized by the total DNA content of KS483 cells after 7 days of culture and expressed as fold induction relative to control. KS483-4C3 cells were stimulated with BMP6, DKK1, and VHH G5 (C) or VHH H7 (D) in a concentration range of 0-70 nM. Coincubation of VHH with DKK1 and BMP6 reversed DKK1-mediated inhibition of BMP6-induced ALP activity in a dose-dependent manner demonstrating effective neutralization of DKK1 activity by the VHH G5 and H7. ALP activity was measured and expressed as relative enzyme activity corrected for DNA and expressed as fold induction relative to BMP6/DKK1/VHH $(+/+/-)$. $* P<0.05$. $(n=3)$. (E) The apparent affinities of different FRZB VHH. Plates were coated with FRZB $(60 \mathrm{nM})$ and blocked with $4 \%$ skimmed milk in PBS (MPBS), then incubated with a concentration range of different VHH $(0-7 \mu \mathrm{M})$. Unbound VHH were washed with PBS-Tween and bound VHH were detected by incubation with mAb M1 and a HRP-conjugated anti-mouse. ALP, alkaline phosphatase; DKK1, Dickkopf 1 homolog; FRZB, frizzled-related protein; HRP, horseradish peroxidase; PBS, phosphate-buffered saline; SD, standard deviation. Color images available online at www.liebertpub.com/scd

FIG. 2. VHH against DKK1 (A) or FRZB (B) can be used for restoring WNT signaling in hChs. A concentration of $5 \mu \mathrm{g} /$ mL DKK1 or FRZB neutralizing antibodies restores WNT activation to its original levels (A, B). The control IgG antibody did not interfere with gene expression in hMSCs (C), hChs (D), and cocultures of hChs and hMSCs (E) of the investigated genes compared to control cells receiving no treatment. hChs, human chondrocytes; hMSCs, human mesenchymal stem cells.
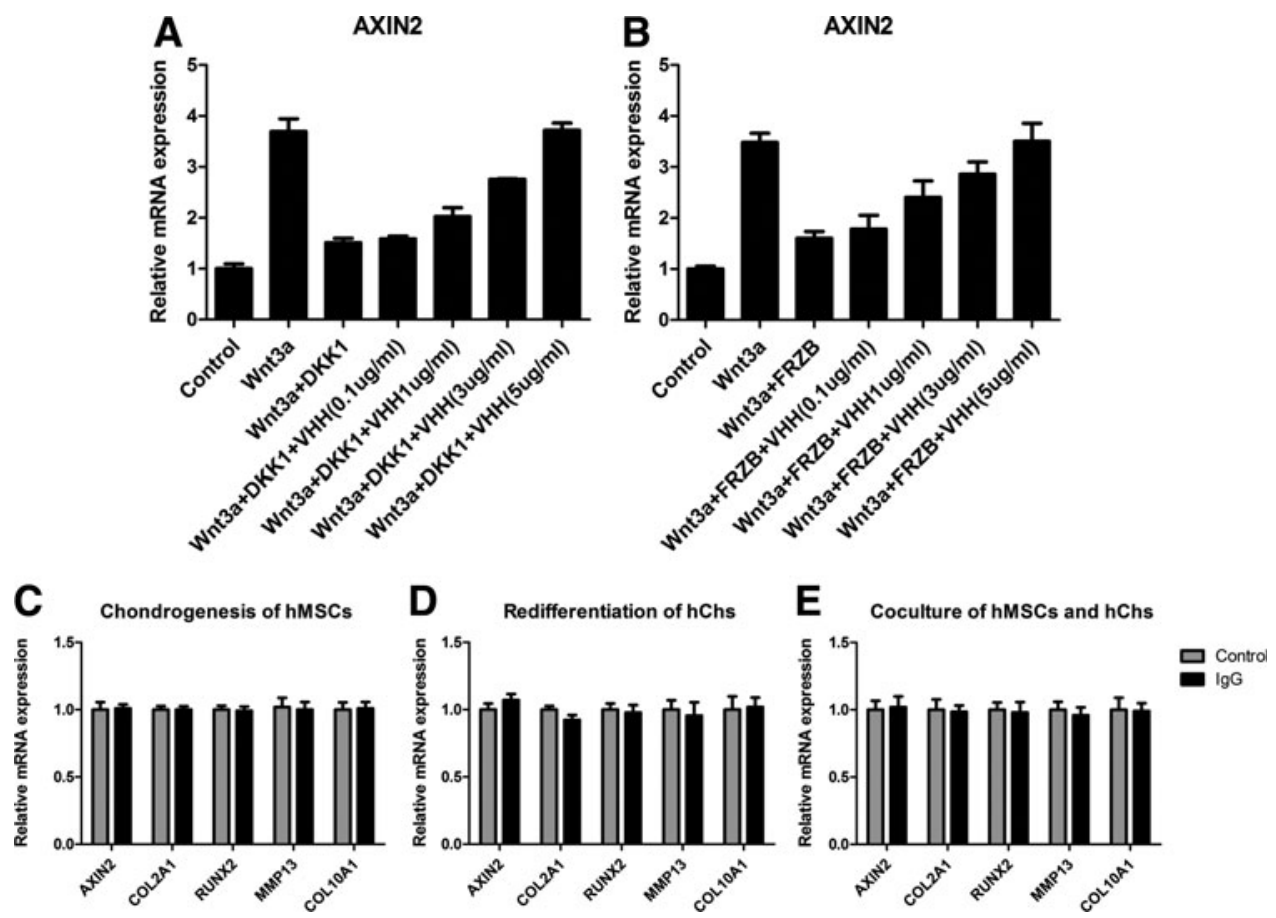
A
GAG

$\overline{\text { Alcian blue Safranin } \mathrm{O}}$
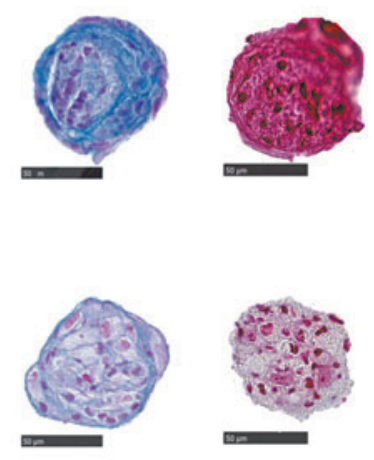

B

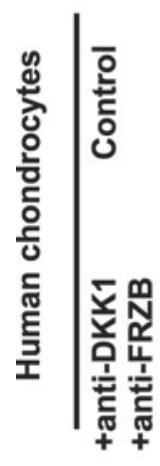

GAG

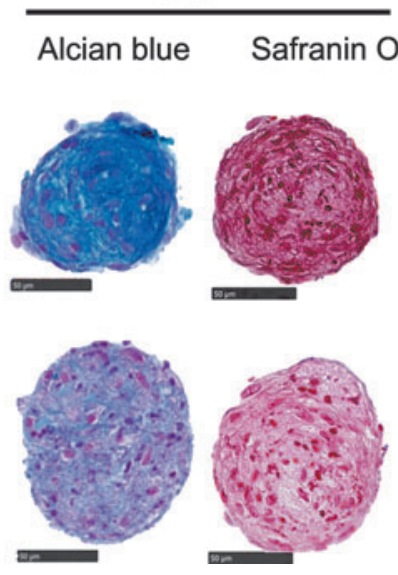

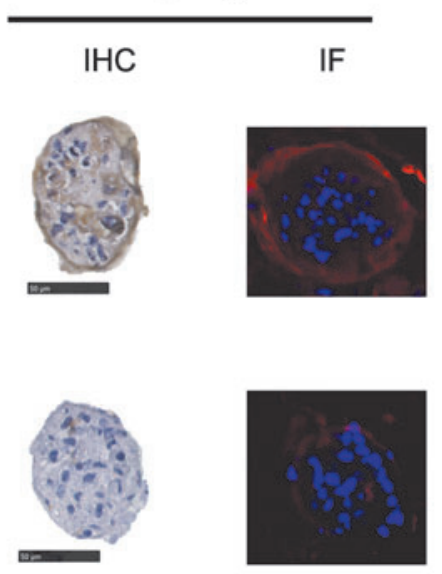

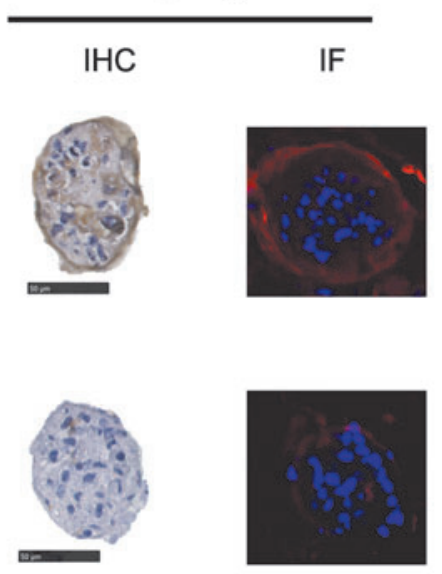

Collagen type II

Collagen type II

FIG. 3. Blocking DKK1 and FRZB inhibits both chondrogenic differentiation of hMSCs and chondrocyte redifferentiation. Histological analysis of GAGs using Alcian blue and safranin $\mathrm{O}$ staining. Collagen type II was measured by IHC and immunofluorescence staining on mid-sagittal sections of the chondrogenically differentiating hMSCs (A) and the chondrocyte (B) micromasses. GAG, glycosaminoglycan; IHC, immunohistochemistry. Color images available online at www .liebertpub.com/scd

The effect of anti-DKK1 and anti-FRZB treatment on chondrogenesis of hMSCs was measured after 4 weeks. We found a significant loss of GAG deposition and the expression of collagen type II in pellets with DKK1 and FRZB blockade compared to the controls. Immunofluorescence further confirmed immunohistochemistry (IHC) result showing the loss of collagen type II (Fig. 3A).

We hypothesized that endogenous expression of DKK1 and FRZB is essential for maintaining the chondrocyte phenotype and that DKK1 and FRZB are necessary for chondrocyte redifferentiation. To test this hypothesis, cells were dedifferentiated during expansion culturing for two passages after which pellet cultures were made to initiate redifferentiation in chondrogenic differentiation medium containing TGF- $\beta$ and DEX. Neutralizing VHH against DKK1 and FRZB were added to the pellet cultures. The effect of anti-DKK1 and anti-FRZB treatment on chondrocyte redifferentiation was measured after 4 weeks. Based on Alcian blue staining, successful redifferentiation of hChs took at least 3 weeks in 3D cultures (Supplementary Fig. S6). Alcian blue and safranin $\mathrm{O}$ showed that GAG deposition was absent in pellets in which DKK1 and FRZB were blocked (Fig. 3B). In addition, blocking DKK1 and FRZB resulted in reduced amounts of collagen type II protein deposition, measured by IHC and immunofluorescence (Fig. 3B).

\section{Blocking DKK1 and FRZB results in expression of hypertrophic markers and cellular hypertrophy in pellet cocultures}

To investigate if endogenous expression of DKK1 and FRZB prevents hypertrophy, neutralizing VHH against DKK1 and FRZB were added to coculture pellets of hMSCs and hChs. Coculture pellets were cultured for 4 weeks in chondrogenic differentiation medium. Gene expression in the cocultures was measured by qPCR. Control experiments using a nonspecific IgG did not affect gene expression (refer to Fig. 2E). Results showed a 1.5-fold increase in mRNA levels of AXIN2 after the addition of the neutralizing VHH, suggesting the activation of WNT signaling after blocking of DKK1 and FRZB (Fig. 4A). Furthermore, blocking DKK1 and FRZB resulted in upregulation of the hypertrophic marker COL1OAl (Fig. 4B) and the master regulator of bone formation $R U N X 2$. MMP13 expression was marginally increased, although significant as shown by $t$-test.

Alcian blue and safranin $\mathrm{O}$ staining indicated that coculturing of hMSCs and hChs in chondrogenic differentiation medium containing neutralizing $\mathrm{VHH}$ showed no obvious difference in GAG production (Fig. 4C). IHC and IF results showed that collagen type II expression had no difference between control and cultures treated with 
A

FIG. 4. The effect of neutralizing antibodies against DKK1 and FRZB on 3D coculture pellets of hMSCs and hChs after 4 weeks of culture. Gene expression was determined by quantitative real-time polymerase chain reaction analysis $(\mathbf{A}, \mathbf{B})$. The expression of target gene was standardized to GAPDH expression. Bars show the mean \pm SD of triplicate cultures. $* P<0.05 ; * * P<0.01$; $* * * P<0.005$ versus control. (C) Paraffin sections of coculture pellets stained for glycosaminoglycan by Alcian blue and safranin $O$. (D) The expression of type II collagen was visualized by IHC and immunofluorescence (scale bar $50 \mu \mathrm{m}$ ). (E) Alizarin red for matrix mineralization (visible as dark red regions) and TUNEL assay for apoptosis (apoptotic nuclei were stained dark brown). (F) Collagen type $\mathrm{X}$ was measured by immunofluorescence. Data are based on eight samples. For each experiment, a representative section is shown. Color images available online at www.liebertpub.com/scd

C

E
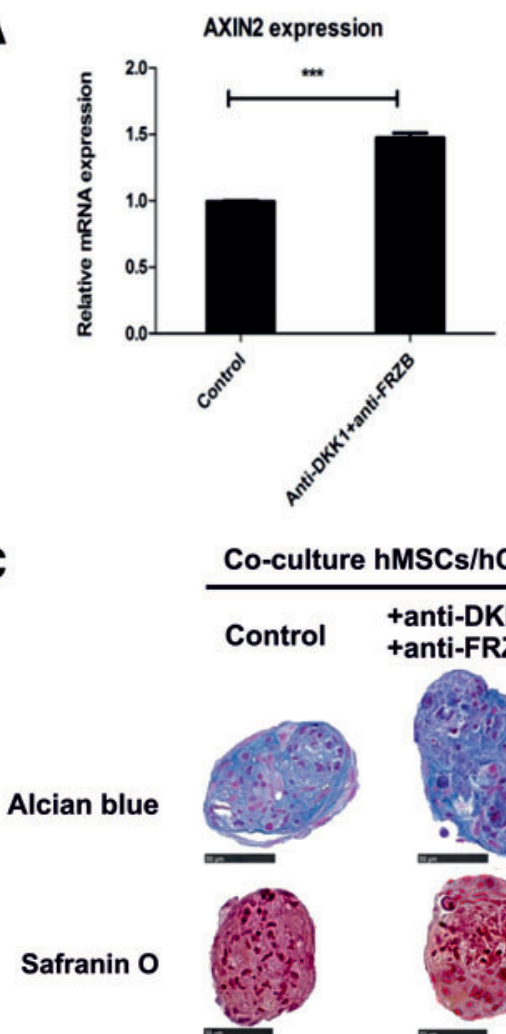

Mineralization

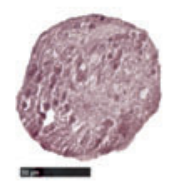

Apoptosis

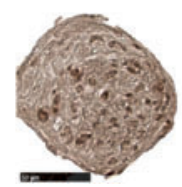

B Hypertrophic markers
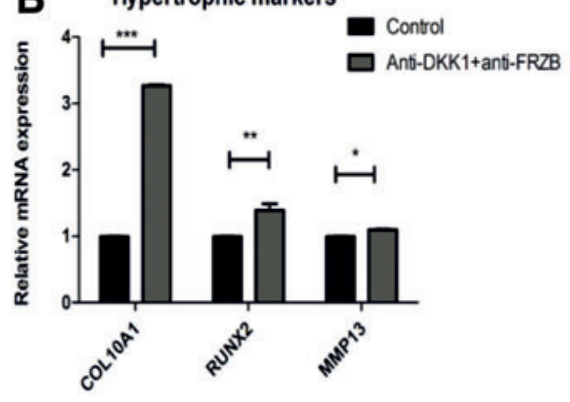

D Co-culture hMSCs/hChs

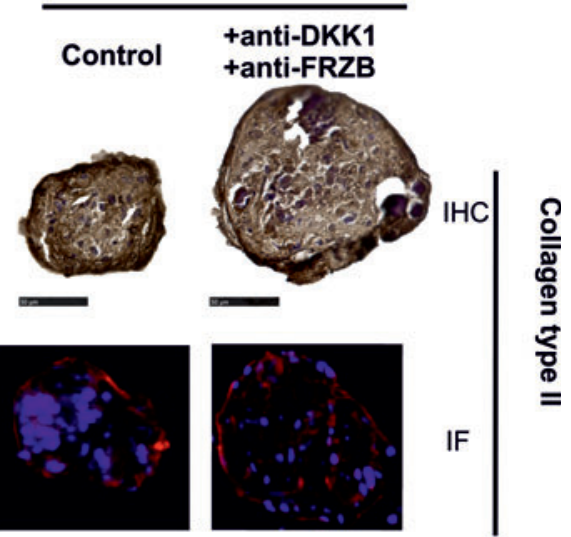

$\mathbf{F}$

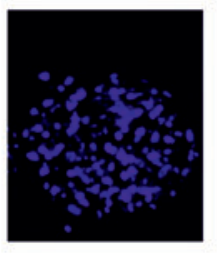

neutralizing VHH (Fig. 4D). In vivo and in vitro, during the process of hypertrophic differentiation, cells enlarge, terminally differentiate, mineralize, and ultimately undergo apoptosis [40,41]. To elucidate the effects of blocking DKK1 and FRZB on (matrix) mineralization and apoptosis, we used Alizarin red $\mathrm{S}$ staining to evaluate mineralization and a TUNEL assay to detect the apoptosis of cells. Alizarin red $\mathrm{S}$ staining indicated that loss of DKK1 and FRZB increased matrix mineralization; the positive staining was especially present in the center of the pellets (Fig. 4E). The TUNEL assay showed that apoptotic cells were present in both the control and the experimental group (Fig. 4E). The number of apoptotic cells was increased in the VHH-treated group. In addition, the coculture pellets treated with neutralizing VHH highly expressed collagen type X (Fig. 4F) and were less compact and more sensitive to tissue handling.

Together, these data suggest that blocking DKK1 and FRZB increases hypertrophic differentiation by increasing hypertrophic marker expression, promotion of cartilage mineralization, and subsequent apoptosis in these cultures.

\section{Discussion}

In this article, we proved that (1) endogenous expression levels of DKK1 and FRZB are necessary for chondrogenesis of hMSCs, (2) endogenous levels of DKK1 and FRZB play a stimulating role in chondrocyte redifferentiation, and (3) endogenous DKK1 and FRZB are able to inhibit hypertrophic differentiation in a coculture system.

Chondrogenesis is defined by a series of steps that can be described as MSC condensation, cell proliferation, and differentiation into chondroprogenitors followed by secretion of ECM components and further maturation into chondrocytes [40] (Fig. 5). WNT/ $\beta$-catenin signaling plays an important role in multiple steps [42] particularly in the initiation of chondrogenesis and inducing hypertrophic differentiation. Both in vivo and in vitro experiments reveal that low levels of $\beta$-catenin activity are required for the initiation of chondrogenesis in the first phase $[43,44]$, while high level of $\beta$-catenin activity blocks the express of cartilage marker by inhibiting the activity of SOX9 [42]. However, the 


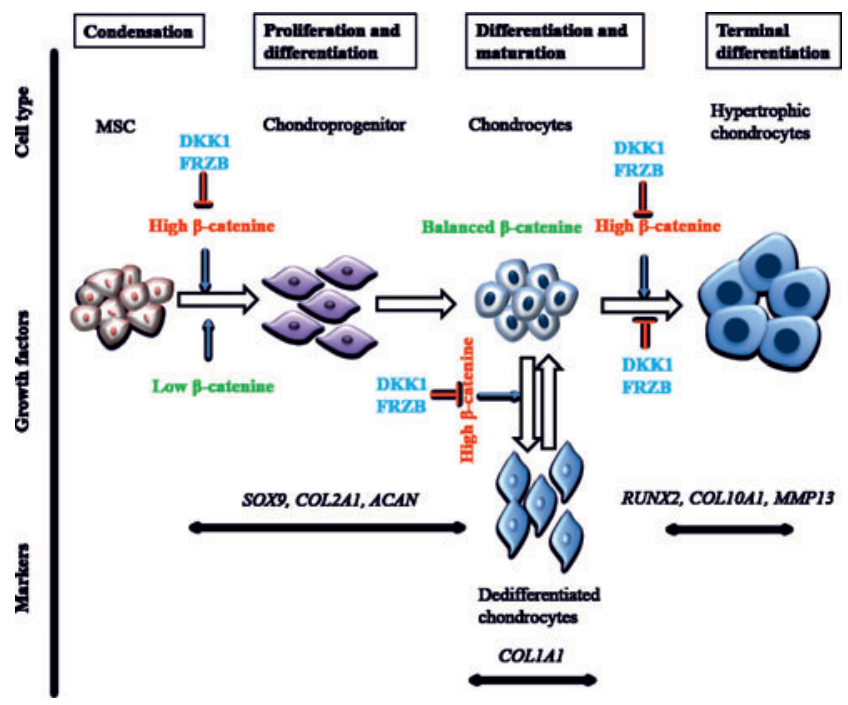

FIG. 5. Endogenously produced WNT antagonists DKK1 and FRZB determine chondrogenesis and chondrocyte terminal differentiation. Chondrogenesis is defined by a series of processes. In the first step of chondrogenesis, DKK1 and FRZB promote chondrogenesis and prevent chondrocyte dedifferentiation through inhibiting WNT/ $\beta$-catenin signaling. In the second step, DKK1 and FRZB inhibit chondrocyte terminal differentiation by blocking WNT/ $\beta$-catenin signaling. The expression of typical markers of the various stages of chondrogenesis is indicated at the bottom. Color images available online at www.liebertpub.com/scd

endogenous mechanism regulating these processes in vivo has not been described. We hypothesized that endogenous expression of DKK1 and FRZB is sufficient to block or repress WNT signaling resulting in initiation of chondrogenesis and prevention of hypertrophic differentiation. Our results show that inhibition of DKK1 and FRZB during chondrogenesis of hMSCs resulted in loss of GAG deposition and collagen type II expression, implying that these factors are indeed necessary for chondrogenic induction of hMSCs.

Primary hChs are enzymatically released from the cartilage matrix for autologous cell-based therapeutic interventions (ACI) [1] and research purposes. However, long expansion time and multiple passaging in monolayer culture, which are generally required to get enough amounts of chondrocytes, lead to dedifferentiation of the isolated chondrocytes. Ryu et al. have reported that $\beta$-catenin expression levels are low in normal chondrocytes and significantly increased as cells undergo dedifferentiation in serial monolayer cultures. They found that overexpression of $\beta$-catenin using a $\beta$-catenin activator leads to dedifferentiation of chondrocytes and that transcriptional activation of $\beta$-catenin is sufficient to cause the loss of chondrocyte phenotype in rabbit cells [45]. In addition, chondrocytes from $A P C$ knockout mice, which display activation of canonical WNT/ $\beta$-catenin signaling, show loss of normal phenotype and dedifferentiation, further proving the role of $\beta$-catenin signaling in chondrocyte dedifferentiation [44].

We proved our hypothesis that DKK1 and FRZB are able to prevent chondrocyte dedifferentiation by blocking WNT/ $\beta$-catenin signaling. Our results showed that antibody-based inhibition of DKK1 and FRZB led to a significant decrease in GAG deposition and the loss of collagen type II, indi- cating that removal of DKK1 and FRZB releases the break on WNT signaling resulting in impaired redifferentiation. We have previously shown that the expression of DKK1 increased (marginally), while FRZB expression decreased during the dedifferentiation process [22].

During dedifferentiation, the small increase in DKK1 might not be sufficient to compensate for the loss of FRZB, which not only blocks canonical but also noncanonical WNT pathways. This is in contrast to DKK1 that only blocks canonical WNT signaling. Loss of FRZB during the dedifferentiation could therefore switch the fine balance between the canonical and the noncanonical WNT pathways. Our data suggest that endogenous expression of DKK1 and FRZB prevents chondrocyte dedifferentiation and that combined blocking of these factors during chondrogenesis prevents redifferentiation.

Loss-of-function and gain-of-function studies indicate that $\mathrm{WNT} / \beta$-catenin signaling is involved in endochondral ossification. Overexpression of $\mathrm{WNT} / \beta$-catenin signaling induces chondrocyte hypertrophy $[42,46]$ while blocking WNT signaling using a WNT inhibitor during the last steps of chondrogenic differentiation prevents hypertrophic differentiation of the cells [23]. We proved our hypothesis that the endogenous WNT inhibitors DKK1 and FRZB play a determining role in preventing terminal chondrocyte differentiation by regulating $\mathrm{WNT} / \beta$-catenin signaling.

In contrast to our monoculture experiments, we found that blocking of DKK1 and FRZB in coculture pellets of hMSCs and hChs did not inhibit cartilage-specific matrix formation and collagen type II production. The discrepancy between monocultures and cocultures suggests that in cocultures other mechanisms are operational that compensate for the loss of DKK1 and FRZB. We have previously shown that MSCs in cocultures secrete trophic factors that enhance cartilage formation and that these factors are not expressed in monocultures of either MSCs or chondrocytes [47]. This implies that in addition to DKK1 and FRZB expression, alternative factors that are specifically present in cocultures compensate for the loss of DKK1 and FRZB, by directly or indirectly regulating WNT activity.

Since we observed GAG and collagen II expression in the cocultures, indicative of cartilage formation, we used the cocultures to study the role of DKK1 and FRZB on terminal chondrocyte differentiation. We observed an increase in hypertrophic markers and matrix mineralization. Matrix mineralization is normally observed in endochondral bone formation [48]. It has been shown that premature induction of hypertrophy during in vitro chondrogenesis of hMSCs correlates with calcification after ectopic transplantion in SCID mice [49]. Both in vivo and in vitro studies have concluded that hypertrophic chondrocytes do not only express hypertrophic markers but also undergo apoptosis [41,49,50]. Similarly, our results showed that the number of apoptotic cells increased in coculture pellets with neutralizing VHH.

In conclusion, as far as we know, our study is the first one to show novel evidence on the combined role of endogenously produced DKK1 and FRZB in at least three stages of chondrocyte differentiation: (1) first, in the initial stage, during onset of chondrogenesis in MSCs, (2) in redifferentiating chondrocytes, and (3) during the hypertrophic differentiation phase (Fig. 5). This study may help us to further improve the clinical cell based cartilage repair strategies. 


\section{Acknowledgment}

L.Z. is funded by the Dutch Arthritis Foundation (Reumafonds) grant number 11-1-408 to J.N.P. and M.K.

\section{Author Disclosure Statement}

No competing financial interests exist.

\section{References}

1. Komarek J, P Valis, M Repko, R Chaloupka and M Krbec. (2010). [Treatment of deep cartilage defects of the knee with autologous chondrocyte transplantation: long-term results]. Acta Chir Orthop Traumatol Cech 77:291-295.

2. Schnabel M, S Marlovits, G Eckhoff, I Fichtel, L Gotzen, V Vecsei and J Schlegel. (2002). Dedifferentiation-associated changes in morphology and gene expression in primary human articular chondrocytes in cell culture. Osteoarthritis Cartilage 10:62-70.

3. Mueller MB and RS Tuan. (2008). Functional characterization of hypertrophy in chondrogenesis of human mesenchymal stem cells. Arthritis Rheum 58:1377-1388.

4. Hubka KM, RL Dahlin, VV Meretoja, FK Kasper and AG Mikos. (2014). Enhancing chondrogenic phenotype for cartilage tissue engineering: monoculture and co-culture of articular chondrocytes and mesenchymal stem cells. Tissue Eng Part B Rev 20:641-654.

5. Green JD, V Tollemar, M Dougherty, Z Yan, L Yin, J Ye, Z Collier, MK Mohammed, RC Haydon, et al. (2015). Multifaceted signaling regulators of chondrogenesis: implications in cartilage regeneration and tissue engineering. Genes Dis 2:307-327.

6. Fischer J, A Dickhut, M Rickert and W Richter. (2010). Human articular chondrocytes secrete parathyroid hormonerelated protein and inhibit hypertrophy of mesenchymal stem cells in coculture during chondrogenesis. Arthritis Rheum 62:2696-2706.

7. Cooke ME, AA Allon, T Cheng, AC Kuo, HT Kim, TP Vail, RS Marcucio, RA Schneider, JC Lotz and T Alliston. (2011). Structured three-dimensional co-culture of mesenchymal stem cells with chondrocytes promotes chondrogenic differentiation without hypertrophy. Osteoarthritis Cartilage 19: 1210-1218

8. Bian L, DY Zhai, RL Mauck and JA Burdick. (2011) Coculture of human mesenchymal stem cells and articular chondrocytes reduces hypertrophy and enhances functional properties of engineered cartilage. Tissue Eng Part A 17: 1137-1145.

9. Yasuhara R, Y Ohta, T Yuasa, N Kondo, T Hoang, S Addya, P Fortina, M Pacifici, M Iwamoto and M EnomotoIwamoto. (2011). Roles of beta-catenin signaling in phenotypic expression and proliferation of articular cartilage superficial zone cells. Lab Invest 91:1739-1752.

10. Zhu M, D Tang, Q Wu, S Hao, M Chen, C Xie, RN Rosier, RJ O'Keefe, M Zuscik and D Chen. (2009). Activation of beta-catenin signaling in articular chondrocytes leads to osteoarthritis-like phenotype in adult beta-catenin conditional activation mice. J Bone Miner Res 24:12-21.

11. Zhu M, M Chen, M Zuscik, Q Wu, YJ Wang, RN Rosier, RJ O'Keefe and D Chen. (2008). Inhibition of beta-catenin signaling in articular chondrocytes results in articular cartilage destruction. Arthritis Rheum 58:2053-2064.

12. Leijten JC, J Emons, C Sticht, S van Gool, E Decker, A Uitterlinden, G Rappold, A Hofman, F Rivadeneira, et al.
(2012). Gremlin 1, frizzled-related protein, and Dkk-1 are key regulators of human articular cartilage homeostasis. Arthritis Rheum 64:3302-3312.

13. Semenov MV, K Tamai, BK Brott, M Kuhl, S Sokol and X He. (2001). Head inducer Dickkopf-1 is a ligand for Wnt coreceptor LRP6. Curr Biol 11:951-961.

14. Bafico A, G Liu, A Yaniv, A Gazit and SA Aaronson. (2001). Novel mechanism of Wnt signalling inhibition mediated by Dickkopf-1 interaction with LRP6/Arrow. Nat Cell Biol 3:683-686.

15. Lin K, S Wang, MA Julius, J Kitajewski, M Moos, Jr. and FP Luyten. (1997). The cysteine-rich frizzled domain of Frzb-1 is required and sufficient for modulation of Wnt signaling. Proc Natl Acad Sci U S A 94:11196-11200.

16. Leyns L, T Bouwmeester, SH Kim, S Piccolo and EM De Robertis. (1997). Frzb-1 is a secreted antagonist of Wnt signaling expressed in the Spemann organizer. Cell 88:747-756.

17. Bafico A, A Gazit, T Pramila, PW Finch, A Yaniv and SA Aaronson. (1999). Interaction of frizzled related protein (FRP) with Wnt ligands and the frizzled receptor suggests alternative mechanisms for FRP inhibition of Wnt signaling. J Biol Chem 274:16180-16187.

18. Kawano Y and R Kypta. (2003). Secreted antagonists of the Wnt signalling pathway. J Cell Sci 116:2627-2634.

19. Gregory CA, AS Perry, E Reyes, A Conley, WG Gunn and DJ Prockop. (2005). Dkk-1-derived synthetic peptides and lithium chloride for the control and recovery of adult stem cells from bone marrow. J Biol Chem 280:2309-2323.

20. Ma B, CA van Blitterswijk and M Karperien. (2012). A Wnt/ beta-catenin negative feedback loop inhibits interleukin-1induced matrix metalloproteinase expression in human articular chondrocytes. Arthritis Rheum 64:2589-2600.

21. Nalesso G, J Sherwood, J Bertrand, T Pap, M Ramachandran, C De Bari, C Pitzalis and F Dell'accio. (2011). WNT-3A modulates articular chondrocyte phenotype by activating both canonical and noncanonical pathways. J Cell Biol 193:551-564.

22. Ma B, JC Leijten, L Wu, M Kip, CA van Blitterswijk, JN Post and M Karperien. (2013). Gene expression profiling of dedifferentiated human articular chondrocytes in monolayer culture. Osteoarthritis Cartilage 21:599-603.

23. Narcisi R, MA Cleary, PA Brama, MJ Hoogduijn, N Tuysuz, D Ten Berge and GJ van Osch. (2015). Long-term expansion, enhanced chondrogenic potential, and suppression of endochondral ossification of adult human MSCs via WNT signaling modulation. Stem Cell Reports 4:459-472.

24. Lories RJ, J Peeters, A Bakker, P Tylzanowski, I Derese, J Schrooten, JT Thomas and FP Luyten. (2007). Articular cartilage and biomechanical properties of the long bones in Frzb-knockout mice. Arthritis Rheum 56:4095-4103.

25. Paalanen MM, E Ekokoski, M El Khattabi, RK Tuominen, CT Verrips, J Boonstra and C Blanchetot. (2011). The development of activating and inhibiting camelid $\mathrm{VHH}$ domains against human protein kinase $\mathrm{C}$ epsilon. Eur $\mathrm{J}$ Pharm Sci 42:332-339.

26. Hoogenboom HR, AP de Bruine, SE Hufton, RM Hoet, JW Arends and RC Roovers. (1998). Antibody phage display technology and its applications. Immunotechnology 4:1-20.

27. Roovers RC, T Laeremans, L Huang, S De Taeye, AJ Verkleij, H Revets, HJ de Haard and PM van Bergen en Henegouwen. (2007). Efficient inhibition of EGFR signaling and of tumour growth by antagonistic anti-EFGR Nanobodies. Cancer Immunol Immunother 56:303-317.

28. Saerens D, J Kinne, E Bosmans, U Wernery, S Muyldermans and K Conrath. (2004). Single domain antibodies 
derived from dromedary lymph node and peripheral blood lymphocytes sensing conformational variants of prostatespecific antigen. J Biol Chem 279:51965-51972.

29. Roovers RC, P Henderikx, W Helfrich, E van der Linden, A Reurs, AP de Bruine, JW Arends, L de Leij and HR Hoogenboom. (1998). High-affinity recombinant phage antibodies to the pan-carcinoma marker epithelial glycoprotein-2 for tumour targeting. Br J Cancer 78:1407-1416.

30. Wesolowski J, V Alzogaray, J Reyelt, M Unger, K Juarez, M Urrutia, A Cauerhff, W Danquah, B Rissiek, et al. (2009). Single domain antibodies: promising experimental and therapeutic tools in infection and immunity. Med Microbiol Immunol 198:157-174.

31. de Marco A. (2011). Biotechnological applications of recombinant single-domain antibody fragments. Microb Cell Fact 10:44.

32. van der Horst G, SM van der Werf, H Farih-Sips, RL van Bezooijen, CW Lowik and M Karperien. (2005). Downregulation of Wnt signaling by increased expression of Dickkopf-1 and -2 is a prerequisite for late-stage osteoblast differentiation of KS483 cells. J Bone Miner Res 20:1867-1877.

33. Verheesen P, A Roussis, HJ de Haard, AJ Groot, JC Stam, JT den Dunnen, RR Frants, AJ Verkleij, C Theo Verrips and SM van der Maarel. (2006). Reliable and controllable antibody fragment selections from Camelid non-immune libraries for target validation. Biochim Biophys Acta 1764:1307-1319.

34. Wu L, X Cai, H Dong, W Jing, Y Huang, X Yang, Y Wu and Y Lin. (2010). Serum regulates adipogenesis of mesenchymal stem cells via MEK/ERK-dependent PPARgamma expression and phosphorylation. J Cell Mol Med 14:922-932.

35. Rivron NC, EJ Vrij, J Rouwkema, S Le Gac, A van den Berg, RK Truckenmuller and CA van Blitterswijk. (2012). Tissue deformation spatially modulates VEGF signaling and angiogenesis. Proc Natl Acad Sci U S A 109:6886-6891.

36. Leijten J, N Georgi, L Moreira Teixeira, CA van Blitterswijk, JN Post and M Karperien. (2014). Metabolic programming of mesenchymal stromal cells by oxygen tension directs chondrogenic cell fate. Proc Natl Acad Sci U S A 111:13954-13959.

37. Muyldermans S, TN Baral, VC Retamozzo, P De Baetselier, E De Genst, J Kinne, H Leonhardt, S Magez, VK Nguyen, et al. (2009). Camelid immunoglobulins and nanobody technology. Vet Immunol Immunopathol 128:178-183.

38. Harmsen MM and HJ De Haard. (2007). Properties, production, and applications of camelid single-domain antibody fragments. Appl Microbiol Biotechnol 77:13-22.

39. Muyldermans S. (2013). Nanobodies: natural singledomain antibodies. Annu Rev Biochem 82:775-797.

40. Zuscik MJ, MJ Hilton, X Zhang, D Chen and RJ O'Keefe. (2008). Regulation of chondrogenesis and chondrocyte differentiation by stress. J Clin Invest 118:429-438.

41. Pacifici M, EB Golden, O Oshima, IM Shapiro, PS Leboy and SL Adams. (1990). Hypertrophic chondrocytes. The terminal stage of differentiation in the chondrogenic cell lineage? Ann N Y Acad Sci 599:45-57.

42. Akiyama H, JP Lyons, Y Mori-Akiyama, X Yang, R Zhang, Z Zhang, JM Deng, MM Taketo, T Nakamura, et al.
(2004). Interactions between Sox 9 and beta-catenin control chondrocyte differentiation. Genes Dev 18:1072-1087.

43. Hill TP, D Spater, MM Taketo, W Birchmeier and C Hartmann. (2005). Canonical Wnt/beta-catenin signaling prevents osteoblasts from differentiating into chondrocytes. Dev Cell 8:727-738.

44. Miclea RL, M Karperien, CA Bosch, G van der Horst, MA van der Valk, T Kobayashi, HM Kronenberg, G Rawadi, P Akcakaya, et al. (2009). Adenomatous polyposis coli-mediated control of beta-catenin is essential for both chondrogenic and osteogenic differentiation of skeletal precursors. BMC Dev Biol 9:26.

45. Ryu JH, SJ Kim, SH Kim, CD Oh, SG Hwang, CH Chun, SH Oh, JK Seong, TL Huh and JS Chun. (2002). Regulation of the chondrocyte phenotype by beta-catenin. Development 129:5541-5550.

46. Pacifici M, E Koyama and M Iwamoto. (2005). Mechanisms of synovial joint and articular cartilage formation: recent advances, but many lingering mysteries. Birth Defects Res C Embryo Today 75:237-248.

47. $\mathrm{Wu} \mathrm{L}, \mathrm{J}$ Leijten, CA van Blitterswijk and M Karperien. (2013). Fibroblast growth factor-1 is a mesenchymal stromal cell-secreted factor stimulating proliferation of osteoarthritic chondrocytes in co-culture. Stem Cells Dev 22:2356-2367.

48. Mackie EJ, L Tatarczuch and M Mirams. (2011). The skeleton: a multi-functional complex organ: the growth plate chondrocyte and endochondral ossification. J Endocrinol 211: 109-121.

49. Pelttari K, A Winter, E Steck, K Goetzke, T Hennig, BG Ochs, T Aigner and W Richter. (2006). Premature induction of hypertrophy during in vitro chondrogenesis of human mesenchymal stem cells correlates with calcification and vascular invasion after ectopic transplantation in SCID mice. Arthritis Rheum 54:3254-3266.

50. Zenmyo M, S Komiya, R Kawabata, Y Sasaguri, A Inoue and M Morimatsu. (1996). Morphological and biochemical evidence for apoptosis in the terminal hypertrophic chondrocytes of the growth plate. J Pathol 180:430-433.

Address correspondence to: Janine N. Post

Department of Developmental BioEngineering Faculty of Science and Technology MIRA-Institute for Biomedical Technology and Technical Medicine University of Twente P.O. Box 217

AE Enschede 7500 The Netherlands

E-mail: j.n.post@utwente.nl

Received for publication July 26, 2016 Accepted after revision September 9, 2016 Prepublished on Liebert Instant Online October 12, 2016 